This document is confidential and is proprietary to the American Chemical Society and its authors. Do not copy or disclose without written permission. If you have received this item in error, notify the sender and delete all copies.

\title{
Sensor Arrays based on Polycyclic Aromatic Hydrocarbons: Chemiresistors versus Quartz-Crystal Microbalance
}

\begin{tabular}{|c|c|}
\hline Journal: & ACS Applied Materials \& Interfaces \\
\hline Manuscript ID: & am-2013-03067t.R1 \\
\hline Manuscript Type: & Article \\
\hline Date Submitted by the Author: & 18-Oct-2013 \\
\hline Complete List of Authors: & $\begin{array}{l}\text { Bachar, Nadav; Technion - Israel Institute of Technology, } \\
\text { Liberman, Lucy; Technion - Israel Institute of Technology, } \\
\text { Muallem, Fairouz; Technion - Israel Institute of Technology, } \\
\text { Feng, Xinliang; Technion - Israel Institute of Technology, } \\
\text { Mullen, Klaus; Max-Planck-Institute for Polymer Research, } \\
\text { Haick, Hossam; Technion - Israel Institute of Technology, Department of } \\
\text { Chemical Engineering }\end{array}$ \\
\hline
\end{tabular}

\section{SCHOLARONE ${ }^{m}$}

Manuscripts 


\title{
Sensor Arrays based on Polycyclic Aromatic Hydrocarbons: Chemiresistors vs. Quartz-Crystal Microbalance
}

\author{
Nadav Bachar, ${ }^{\perp}$ Lucy Liberman, ${ }^{\perp}$ Fairouz Muallem ${ }^{\perp}$, Xinliang Feng,${ }^{\S}$ \\ Klaus Müllen, ${ }^{\S}$ and Hossam Haick ${ }^{*},{ }^{\perp}$ \\ $\perp$ The Department of Chemical Engineering and Russell Berrie Nanotechnology Institute, Technion- \\ Israel Institute of Technology, Haifa 3200003, Israel \\ \& Max-Planck-Institute for Polymer Research, Postfach 3148, D-55021 Mainz, Germany
}

\section{CORRESPONDING AUTHOR: Hossam Haick}

Department of Chemical Engineering and Russell Berrie Nanotechnology Institute, Technion-Israel Institute of Technology, Haifa 3200003, Israel.

Tel: +972(4)8293087; Fax: +972-77-8871880; E-mail: hhossam@technion.ac.il 


\begin{abstract}
Arrays of broadly cross-reactive sensors are key elements of smart, self-training sensing systems. Chemically sensitive resistors and quartz-crystal microbalance (QCM) sensors are attractive for sensing applications that involve the detection and classification of volatile organic compounds (VOCs) in the gas phase. Polycyclic aromatic hydrocarbon (PAH) derivatives as sensing materials can provide good sensitivity and robust selectivity towards different polar and nonpolar VOCs, while being quite tolerant to large humidity variations. Here, we present a comparative study of chemiresistor and QCM arrays based on a set of custom-designed PAH derivatives having either purely nonpolar coronas or alternating nonpolar and strongly polar side chain termination. The arrays were exposed to various concentrations of representative polar and nonpolar VOCs under extremely varying humidity conditions $(5-80 \% \mathrm{RH})$. The sensor arrays' classification ability of VOC polarity, chemical class and compound separation was explained in terms of the sensing characteristics of the constituent sensors and their interaction with the VOCs. The results presented here contribute to the development of novel versatile and cost-effective real-world VOC sensing platforms.
\end{abstract}

KEY WORDS: Polycyclic Aromatic Hydrocarbon; volatile organic compound; polarity; sensor array; chemiresistor; quartz-crystal microbalance 


\section{INTRODUCTION}

Arrays of broadly cross-reactive sensors have received significant attention as key elements of smart, self-training sensing systems. ${ }^{1-5}$ These arrays could meet the growing demand for rapid and flexible online detection of a wide range of chemical and biological agents in different branches of industry, homeland security, 5, 6 environmental monitoring, 5, 7,8 and medical diagnostics $^{1-5,9-20}$. Combined with statistical algorithms, these sensor arrays can provide convenient bionics of the mammalian olfactory system for identifying complex gas samples, without the necessity of identifying their separate ingredients. ${ }^{1-5}$ In this configuration, every sensing element responds to all or to part of the chemical compounds in the gas sample. Odorspecific response patterns are then established by applying pattern recognition- and classification algorithms. ${ }^{21,22}$ A sensor array can be trained for the selective detection of a specific odor by repeated exposure. Thereafter, unknown samples can be identified by comparison with the patterns stored in a database.

When using this strategy, the sensing platforms and sensing materials must be prudently chosen to ensure sufficient detection limits and dynamic ranges for the chemical compounds that cause the odors of interest. ${ }^{1,3}$ Additionally, it has to be chosen with tolerance to variable background parameters that typically occur in real-world applications. ${ }^{1,3,5}$ For example, varying levels of ambient humidity usually alter the sensing signals, leading to a decrease in sensitivity and selectivity towards the desired compound(s). ${ }^{12} \quad 23$ The implementation of the sensors should be simple and cost-effective enough to be economically viable for real-world applications. Sensors with electronic and electro-acoustic transduction mechanisms, such as chemically sensitive resistors ${ }^{24-33}$ and quartz-crystal microbalance (QCM) sensors $^{34-36}$ are among the most attractive and widespread elements for sensing applications that involve the detection and classification of volatile organic compounds (VOCs) in the gas phase..$^{9,11,13,16,19,20,24,37}$ 
Recently, we have shown that polycyclic aromatic hydrocarbon (PAH) derivatives as sensing materials in chemiresistors, field effect transistors or QCM sensors can provide good sensitivity and robust selectivity towards different polar and nonpolar VOCs, while being highly tolerant even to drastic humidity variations. ${ }^{11,33,34,38-40}$ For example, we have shown that a bilayer structure with a quasi $2 \mathrm{D}$ network of single wall carbon nanotubes (RNSWCNTs) as an under-layer and a micron-thick PAH film as an overcoat, can provide excellent detection and classification between polar and nonpolar VOCs, both in dry atmospheres with $\sim 5 \%$ relative humidity $(\mathrm{RH})$ and in almost fully humidified atmospheres $(\sim 80 \% \mathrm{RH}) .^{33,34,38}$ Electrical current flowing through the structure favors the low resistance RN-SWCNTs, and, therefore, the PAH films serve mainly as an adsorption phase for the targeted VOCs. ${ }^{33,38-40}$ Nevertheless, the interaction between the VOCs the chemical/physical properties of the PAH sensing materials, as well as their incorporation into arrays of chemiresistors and QCM sensors still need further exploration.

Here, we present a comparative study of the sensing performance of six customdesigned PAH derivatives having purely nonpolar alkyl side chains or alternating nonpolar and strongly polar side chain termination. The PAH layers were applied as sensing elements in PAH-functionalized RN-SWCNT chemiresistors and in PAH-coated QCM sensors. The sensing responses to representative polar and nonpolar VOCs from the families of alcohols, alkanes, ethers and aromatics were studied at a range of concentrations and under extremely varying humidity conditions, from dry to almost fully humidified atmospheres. The classification ability of VOCs polarity, chemical class and compound separation was determined for chemiresistor arrays and QCM sensor arrays incorporating the same set of sensing materials and was explained in terms of the sensing characteristics of the constituent sensors. Sub-arrays containing either PAH with nonpolar alkyl side chains or PAHs 
containing alternating nonpolar and strongly polar end groups were tested and compared in order to gain better insight into the interaction between VOCs and PAH sensing elements.

\section{EXPERIMENTAL SECTION}

\section{Device preparation}

The properties (core and side-chains) and the widely modified molecular structures of the six PAH derivatives that were used in this study are listed in Table 1. The cores are hydrophobic mesogens, either aromatic cores with a carbon number of 42 (PAH-B, PAH-C), semi-triangle shaped aromatic cores with a carbon number of 48 (PAH-3, PAH-4 and PAH-5), or a triangleshaped core with a carbon number of 60 (PAH-A). The side chains are different straight and branched alkyl chains that are in part terminated with functional substituents, such as ester (PAH-3) and carboxylic acid (for PAH-4 and PAH-5). The synthesis of the three PAH derivatives with nonpolar side-chains (PAH-A, PAH-B, PAH-C) has been described in Refs. 41, 42. The synthesis of the three PAH derivatives with alternating nonpolar and strongly polar side-chains (PAH-3, PAH-4, and PAH-5) will be described elsewhere. $10^{-3} \mathrm{M}$ PAH solutions were prepared in toluene (PAH-A; PAH-C; and PAH-3), in tetrahydrofuran (PAH-4; and PAH-5) or in xylene (PAH-B). PAH molecules easily self-assemble into long molecular stacks with a large, electron rich, semiconducting core, providing good charge carrier transport along the molecular stacking direction and a relatively insulating periphery. ${ }^{41,43-45}$ The molecular PAH columns typically form micrometer sized, and sponge-like 3D structures with a high surface-to-volume ratio. ${ }^{33,39,40}$

Mass-sensitive sensors were prepared by drop-casting $2 \mu \mathrm{L}$ of PAH solution on goldplated Quartz Crystal Microbalance (QCM) resonators. ${ }^{33,34,39,40,46}$ The devices were dried for 30 min under ambient conditions, then placed into a desiccator and kept overnight under vacuum. Chemiresistors were prepared from hybrid layers of quasi-2D random networks of 
single walled carbon nanotubes (RN-SWCNTs) that were capped with micron-thick PAH layers, as described previously. ${ }^{33,} 39,40$ Briefly, 10 pairs of circular interdigitated (ID) electrodes (outer diameter: $3000 \mu \mathrm{m}$; electrode spacing \& electrode width: $25 \mu \mathrm{m}$ ) were deposited on substrates (p-type Si (100) with a $1 \mu \mathrm{m}$ thermal oxide), by electron-beam evaporation of $25 \mathrm{~nm} / 200 \mathrm{~nm} \mathrm{Ti} / \mathrm{Au}$, using conventional photolithography. Electrically continuous RN-SWCNTs were prepared by drop-casting $0.02 \%$ wt SWCNT solution (ARRY International LTD, Germany; average diameter: $1.5 \mathrm{~nm}$; average length: $7 \mu \mathrm{m} ; \sim 30 \%$ metallic/70\% semiconducting) in dimethylformamide (DMF; Sigma Aldrich Ltd.; $>98 \%$ purity) onto the ID electrodes. The RN-SWCNT devices were dried overnight in a vacuum oven and subsequently coated with chemiresistive PAH layers by drop-casting $2 \mu \mathrm{L}$ of solution. The PAH/RN-SWCNT chemiresistors were dried under ambient conditions for 30 min, subsequently placed in a desiccator and kept under vacuum overnight. The PAH deposition process was twice repeated to obtain nearly continuous PAH cap-layers, as in the QCM devices. The morphology of the PAH films in both chemiresistors and QCM devices were similar to each the other and were similar to those presented and discussed by us in an earlier report. ${ }^{38}$

\section{Description of the Sensor Arrays}

Two sensor arrays were studied. The constituent sensors of these arrays are summarized in the Table S1 in the supporting information. Array 1 incorporated six PAH/RN-SWCNT chemiresistors. Array 2 consisted of six PAH-functionalized mass sensitive QCM devices. Each of the six constituent sensors was functionalized with one of the six PAH derivatives listed in Table 1; both arrays included all of the six available PAH derivatives. The PAH derivatives formed two groups (see Table 1): (i) PAH-A,B\&C had different poly-aromatic cores and different nonpolar hydrophobic alkyl side-chains; (ii) PAH-3,4\&5 had the same 
semi-triangular poly-aromatic cores (carbon number: 48) and different side chains: alternating nonpolar hydrophobic alkyl side-chains and strongly polar hydrophilic side-chains terminated with different polar functional substituents.

Array 1 was characterized by electrical measurements before and after exposure to a series of polar and nonpolar VOCs (see Table 2) at various concentrations and under different relative humidity $(\mathrm{RH})$ backgrounds. Array 2 was characterized by mass absorption measurements under the same exposure conditions (see below).

\section{Exposure to VOC Vapors}

Table 2 lists the polar and nonpolar VOCs that were used in this study: DI-water from a commercial water purification system (Easypure II); 1-decanol, dibutylether and mesitylene (all from Sigma-Aldrich Ltd.); 1-hexanol and 1-octanol (from Fluka Analytical Ltd), ethanol and $n$-hexane (from Frutarom Ltd.); $n$-decane (from Arcos Organics); $n$-octane (from Merck Schuchardt Ltd). Purity of all VOCs $>99 \%$.

VOC vapors were supplied to the sensors using an automated, computer-controlled flow system, capable of regulating the VOC concentrations and the background humidity level. Oil-free, purified air from a compressed air source (baseline RH: 5.0 $0.2 \%$; organic contamination determined by a commercial PID ppbRAE 3000 detector $<0.3 \mathrm{ppm}$ ), was used as a carrier gas and as a reference gas. The VOC or water vapours were produced by bubbling air through liquid VOCs in glass bubblers. Saturated VOC vapors were carried out from the side arm of the bubbler and diluted with a carrier gas at a flow rate of $0.5-5.0 \mathrm{~L} / \mathrm{min}$. By regulating the flow between the saturated VOC vapor and the gas carrier, various concentrations $\left(p_{a} / p_{o}=0.05\right.$ to 0.2 , where $p_{a}$ stands for the partial pressure of the VOC and $p_{o}$ stands for the saturated vapor pressure at $\left.21^{\circ} \mathrm{C}\right)^{47}$ were achieved. The background relative humidity $(\mathrm{RH} ;$ i.e. the ratio of the partial pressure of water vapor in the air-water mixture to 
the saturated vapor pressure of water at the prescribed temperature) of the carrier gas was regulated by a deliberate mixing of dry air $(5.0 \pm 0.2 \% \mathrm{RH})$ and fully humidified air $(100 \%$ $\mathrm{RH}$ ) coming out of the water bubbler) flows. Three main background $\mathrm{RH}$ were used: $5 \%$, $40 \%$ and $80 \% \mathrm{RH}$.

Sensing experiments were performed continuously, using 9 subsequent exposure cycles in a flow mode with incremental increase of the VOC concentration: (i) exposure to synthetic dry air flow with $\mathrm{RH}=5 \%$ for $10 \mathrm{~min}$; (ii) exposure to $\mathrm{VOC}$ vapor at $p_{a} / p_{o}=0.05$ at a fixed background RH for $10 \mathrm{~min}$; (iii) purging the chamber with dry air for $10 \mathrm{~min}$; (iv) exposure to VOC vapor at $p_{a} / p_{o}=0.10$ (fixed $\mathrm{RH}$ background) for $10 \mathrm{~min}$; (v) purging the chamber with dry air for $10 \mathrm{~min}$; (vi) exposure to VOC vapor at $p_{a} / p_{o}=0.16$ (fixed RH background) for $10 \mathrm{~min}$; (vii) purging the chamber with dry air for $10 \mathrm{~min}$; (viii) exposure to VOC vapor at $p_{a} / p_{o}=0.2$ (fixed RH background) for $10 \mathrm{~min}$; (ix) purging the chamber with dry air for 10 min. $^{47}$ These 9 exposure cycles were performed for three fixed background RH levels of $5 \%, 40 \%$ and $80 \%{ }^{48}$ Every experiment was run twice to test reproducibility. Array 1 was characterized by electrical measurements during the multi-cycle exposures to a series of polar and nonpolar VOCs (see below). Array 2 was characterized continuously by mass absorption measurements under the same exposure conditions (see below).

\section{Electrical Measurements}

The six PAH/ RN-SWCNT chemiresistors were integrated into TO5 holders by wire bonding. The bonded devices were then mounted on a custom circuit board with twenty separated sensor sites and were horizontally inserted into a $350 \mathrm{ml}$ stainless steel exposure chamber. Both gas inlet and outlet were parallel to the sensors stage in the chamber. The changes in resistance under VOC exposure were measured using the Agilent multifunction switch 34980. A Stanford Research System SR830 DSP lock-in amplifier controlled by an IEEE488 bus 
was used to supply the AC voltage signal $(0.2 \mathrm{~V}$ at $1 \mathrm{kHz})$ and to measure the corresponding current $(<10 \mu \mathrm{A}$ in the studied devices). The entire system was controlled by a custom Labview program. The relative resistance change, $\Delta R / R_{b}$, was determined from the measured data (see supporting information, Figure $\mathrm{S} 1 \mathrm{~b})$, where $\Delta R$ is the resistance change $\left(R-R_{b}\right)$ of the film upon exposure to VOC and $R_{b}$ is the films baseline resistance in dry air prior to the exposure.

\section{Mass Absorption Measurements}

QCM sensors are piezoelectric mass-sensing devices that can detect very small mass changes, down to a fraction of a monolayer or a single atomic layer. A QCM sensor measures the change in frequency $(\delta f)$ of a quartz crystal resonator resulting from the addition or removal of a small mass $(\Delta m)$ at the surface of the acoustic resonator. $\Delta m$ can be determined from $\delta f$ using the Sauerbrey's equation, $\Delta m=-C_{f} A \cdot \delta f ; C_{f}$ : mass sensitivity (here: $1.104 \mathrm{ng} \cdot \mathrm{cm}^{-}$ $\left.{ }^{2} \cdot \mathrm{Hz}^{-1}\right) ; A$ : active area of the QCM resonator (here: $0.2 \mathrm{~cm}^{2}$ ). The frequency shifts $(\delta f)$, before and after exposure to VOC vapors were recorded simultaneously for the six PAH-QCM sensors, using a LibraNose 2.1 system (Technobiochip, Elba Island, Italy). This QCM system incorporates a stainless steel exposure chamber with eight QCM mounts that are located in parallel to gas flow, an internal micro-pump and a micro-electric valve that conveys the VOC vapors to the exposure chamber with the sensors. The gas inlet entered from the bottom of the chamber and the outlet stream exited from its top. The relative frequency shift, $\Delta f / f_{o b}$, was determined from the measured data (see supporting information, Figure S1a), where $\Delta f$ is the frequency change $\left(f-f_{b}\right)$ of the device upon exposure to VOC and $f_{b}$ is the baseline frequency. The baseline normalizations were carried out in order to remove any variability due to the use of different film thickness and/or mass between experiments on a given type of film. The results were given as relative mass change, $\Delta m / m_{b}$. 


\section{Statistical Analysis of the Sensors Array Output}

Collective patterns were derived from the output of the sensor arrays, using principle component analysis (PCA) and discriminant factor analysis (DFA) ${ }^{49} \mathrm{PCA}$ is a non-supervised linear multivariate analysis method which reduces the complexity of the data-set, from initial n-dimensional space to a few dimensions. This is done using a linear combination of the original measured variables resulting in new uncorrelated (orthogonal) variables called principle components (PCs). These PCs allow preserving the date-set inherent structure while its resulting variance is maximized. PCA is performed with no information on the classification of samples and it is based solely on the intrinsic variance of the original data set. On the other hand, DFA is a supervised linear multivariate analysis method that is supplied with the classification information regarding every measurement in the training set. DFA finds new orthogonal axes (canonical variables) as a linear combination of the input variables, computing these factors for minimizing the intra-class variance while maximizing the interclass variance. The classification accuracy is determined by leave-one-out cross-validation. Given $n$ measurements, DFA is computed $\mathrm{n}$ times using $n-1$ training vectors. The left out vector (i.e. the validation vector that is invisible during the training phase) is then projected onto the DFA model, producing a classification result. The classification accuracy is estimated as the averaged performance over the $\mathrm{n}$ tests. The statistical tests were performed by SAS JMP, ver. 9.

\section{RESULTS AND DISCUSSION}

\section{Response Characteristics of PAH/RN-SWCNT Chemiresistors and PAH-QCM}

Each of the twelve tested sensors in array $1 \&$ array 2 (see supporting information, Table S1) responded rapidly and reproducibly to the exposure of the polar and nonpolar VOCs that are listed in Table 2, at the studied concentrations between $p_{a} / p_{o}=0.05$ and $p_{a} / p_{o}=0.2$, and under 
different $\mathrm{RH}$ background levels of $5 \%, 40 \%$ and $80 \%$. All signals were well above the detection- and quantification limits of the sensors. Two representative examples of the recorded net sensing responses (of the PAH-4 QCM sensor and the PAH-4/RN-SWCNT chemiresistor during one complete hexane cycle at $5 \% \mathrm{RH}$ ), are given in the supporting information, Figure S1.

Negative frequency shifts were observed for all the PAH-coated QCM sensors upon exposure to the VOCs, corresponding to an increase in the PAH films' mass. This mass increase could be due to (i) dissolution of VOC molecules into the PAH layer, and/or (ii) accumulation of VOC molecules on top of the PAH layer surface. Positive resistance shifts were observed for all six PAH/RN-SWCNT chemiresistors, corresponding to a decrease in the PAH films' conductivity. When switching off the vapor supply and purging the exposure chamber with dry air, the QCM sensors returned rapidly to their baseline frequencies. The baseline shifts were negligible. The chemiresistors showed a small and constant drift in baseline resistance with time. The changes in the electrical resistance and resonant frequency that can be observed in Figure 1S of the supporting information were caused exclusively by exposure to the VOC/water vapors since the total flow rate, $\mathrm{RH}$ level $( \pm 3 \%)$ and temperature $\left( \pm 2{ }^{\circ} \mathrm{C}\right)$ were maintained constant throughout the experiments. Evidently, as the VOC concentration increased, the responses, in their absolute value, increased as well. For the sake of comparative analysis, we extracted the relative responses in the form of $\Delta R / R_{b}$ and $\Delta m / m_{b}$ form the measured time-dependent sensing responses of the chemiresistors and of the QCM sensors, respectively.

The sensing properties of PAH-films are primarily determined by the properties of the side chains of the PAH molecules. Figure 1 compares the performance as sensing matrix in chemiresistors and QCM devices of a PAH derivative with only nonpolar side-chains to that of a PAH derivative having strongly polar outer termination. PAH-A with branched alkyl 
chains was taken as a representative example of the former, and PAH-4 with alternating branched alkyl and carboxyl terminated phenyl side-chains served as representative example for the latter.

Figures $1(\mathrm{a} \& \mathrm{~b})$ show $\Delta R / R_{b}$ of the PAH-A/RN-SWCNT and PAH-4/RN-SWCNT chemiresistors $v s$. the VOC concentration $\left(p_{a} / p_{o}\right)$ for all studied VOCs at $5 \%$ background RH. Figures $1(\mathrm{c} \& \mathrm{~d})$ show the concentration dependence of $\Delta m / m_{b}$ for QCM devices incorporating the same PAH films under identical conditions. It was observed that for all tested VOCs both $R / R_{b}$ and $\Delta m / m_{b}$ increased in good approximation linear with increasing VOC concentrations $\left(\mathrm{R}^{2}>0.9\right)$ within the tested range of concentrations $\left(p_{a} / p_{o}=0.05-0.2\right)$. The chemiresistors exhibit good VOC separation of similar magnitude over the entire range of compositions for both PAH molecules, while the signals of the QCM devices overlapped strongly at $p_{a} / p_{o}=0.05$, but fanned out at higher concentrations. This trend was more pronounced for PAH-A than for PAH-4.

Both PAH-A and PAH-4 showed lower uptake of the polar VOCs than of the nonpolar ones (see Figure $1 \mathrm{c} \& \mathrm{~d}$ ); the mass uptake of PAH-A was almost negligible and smaller than that of PAH-4, in agreement with our earlier studies. ${ }^{34}$ However, the relatively small mass changes after polar VOC exposure were sufficient to produce significant changes in the electrical resistance both for PAH-A and PAH-4. In contrast, the nonpolar VOCs yielded significant mass uptakes correlated with relatively low changes in $\Delta R / R_{b}$ (see Figure $1 \mathrm{a} \& \mathrm{~b}$ ). Remarkably, the uptake of polar molecules and water was smaller for PAH-A than for PAH4, while their resistance responses were similar. Hence, the difference in sensing performance between the QCM devices and the chemiresistors must be more pronounced for the PAH-A macromolecule with the nonpolar side-chains than for PAH-4 with mixed nonpolar and polar side chains. This could be observed clearly when plotting $\Delta R / R_{b} v s . \Delta m / m_{b}$ (see Figure 1 e\&f). For PAH-A, the curves for the polar VOCs had a very steep increase of $\Delta R / R_{b}$ within a very 
small $\Delta \mathrm{m} / \mathrm{m}_{b}$ interval and remained close to the $\Delta R / R_{b}$-axis, while the curves for nonpolar VOCs were shallow and displayed only a moderate increase of $\Delta R / R_{b}$ with increasing mass uptake. PAH-4 showed a similar but considerably less pronounced trend.

The change in the slopes of $\Delta R / R_{b} v s . \Delta \mathrm{m} / \mathrm{m}_{b}$ was illustrated in Figures $1 \mathrm{~g} \& \mathrm{~h}$ by plotting their ratio against the VOC concentration for PAH-A and $\mathrm{PAH}-4$, respectively. For the nonpolar VOCs, the ratios of $\Delta R / R_{b}$ and $\Delta m / m_{b}$ were almost constant and close to 1 over the entire range of VOC concentrations. This trend was observed both for PAH-A and PAH-4. The good agreement between mass uptake and resistance response could possibly indicate the following: the nonpolar VOCs causing the observed mass increase of the PAH layer were mostly dissolved into the layer (and did hardly accumulate on top of the layer surface), thus causing a corresponding volume change or fractional swelling of the PAH film, which in turn disrupted the underlying RN-SWCNTs, thus leading to the observed proportional increase in the electrical resistance. However, further investigations of the morphological changes of the PAH layer under VOC exposure would be necessary before far-reaching conclusion can be drawn. In contrast, a strong discrepancy was observed for polar VOCs at low concentrations between resistance response and mass uptake. This trend was most pronounced for all the polar VOCs (except for water) at the lowest VOC concentration of $p_{a} / p_{o}=0.05$. Again, the discrepancies were more obvious for PAH-A $\left(\Delta R / R_{b}\right.$ up to two orders of magnitude higher than $\Delta m / m_{b}$ ) than for PAH-4 (about 1 order of magnitude difference). The discrepancies decreased monotonically, in a linear way, for increasing VOC concentrations, but still remained higher than for the nonpolar VOCs over the entire range of concentrations. We propose the following educated guess about the possible underlying physical mechanism: only part of the polar VOCs could be dissolved into the PAH layer, while the rest remained on the surface, forming VOC accumulation layer. ${ }^{50,51}$ It would be reasonable to assume that at low VOC concentrations the proportion of VOC molecules entering the PAH layer would be 
higher than at higher concentrations, due to saturation. ${ }^{52-54}$ However, as mentioned before, detailed morphological studies under VOC exposure would be necessary to confirm this hypothesis. For pure water vapor at concentrations of $p_{a} / p_{o}=0.05-0.2$, the relative resistance response $\Delta R / R_{b}$ of PAH-A was about 30 times higher than relative mass uptake $\Delta m / m_{b}$, while $\Delta R / R_{b}$ of PAH-4 was only about 5 times higher than $\Delta m / m_{b}$. Clearly, the sensing mechanism for polar VOCs is more complex than for nonpolar VOCs and most probably results from an interplay of different physical mechanisms for the interaction of the polar VOCs with the PAH layer that are related to charge exchange and/or dielectric effects. This may include, but are not limited to: (a) a decrease in the electron hopping rate in the film caused by an increase in the dielectric constant of the film's medium ${ }^{50}$ due to the incorporation of polar molecules having a high dielectric constant than the PAH host layer (see Table 2); and/or (b) a reduction in the concentration of the charge carriers and, hence, in the conductivity, of the PAH layer, probably due to electron withdrawal by the electronegative oxygen atom in the hydroxyl group attached to the saturated carbon in the polar molecule. ${ }^{55}$

Similar trends were observed for all six PAH derivatives, both as chemiresistors and as QCM sensors, irrespective of the humidity background levels. The heat-map representation in Figure 2 summarizes the relative resistance changes for the six studied PAH derivatives to polar and nonpolar VOCs at concentrations between $p_{a} / p_{o}=0.05$ and $p_{a} / p_{o}=0.2$ and under low $(5 \%)$, intermediate $(40 \%)$ and high $(80 \%)$ background $\mathrm{RH}$ levels. The corresponding relative mass changes are summarized in Figure 3. We assume that the sensing of polar and nonpolar VOCs by the PAH films is related to molecular interactions such as dipole interactions and van der Waals forces between the VOCs and PAH macromolecules, as well as to the film's microstructure. At the molecular level, we assume parameters like core size and shape, conjunction length, side chain nature and length and intermolecular forces (such as hydrogen bonding, ${ }^{46,56}$ van der Waals and aromatic/ $\pi$-stacking ${ }^{57}$ ) can dominate individually or 
cooperatively to establish the organization of the system, thus may influence the charge transport. At the macrostructure level, we assume that parameters like film morphology, distribution of delocalized hopping states and density and height of grain boundary barrier ${ }^{58}$ may also affect the conductivity. The interactions between VOCs and sensing film most likely modulate one or more of these parameters, thus regulating the current density through the film.

The following observations emerged from a comprehensive comparative analysis of the sensing signals:

(i) The responses of all chemiresistors and QCM sensors increased systematically as higher VOC concentrations were introduced. The increase of the sensing response was in good approximation proportional (linear) to the increasing VOC concentration.

(ii) All PAH/RN-SWCNTs chemiresistors exhibited a higher resistance increase to ethanol than to the other VOCs. Among the nonpolar VOCs, the highest resistance increase was observed for decane.

(iii) All PAH-coated QCM sensors showed the highest mass increase when exposed to the aromatic compound mesitylene. Indeed, mesitylene can act as a solvent for the PAH macromolecules. It is reasonable to assume that aromatic-aromatic interactions and/or van der Waals forces between the fused benzene 1 rings in the PAH cores and those of the mesitylene occur that cause the enhanced mass uptake.

(iv) In most cases the PAH-A,B\&C/RN-SWCNTs chemiresistors, which possess only hydrophobic alkyl side-chains, showed lower resistance increases than the PAH3,4\&5/RN-SWCNTs, which possess alternating hydrophobic alkyl side-chains and hydrophilic side-chains terminated with different polar functional substituents. The same was observed for the PAH-coated QCM sensors. 
(v) In all PAH/QCM sensors the mass adsorption of nonpolar VOCs was higher than that of the polar VOCs, while in most PAH/RN-SWCNTs sensors the polar VOCs exhibited higher increases in resistance than the nonpolar VOCs, with the exception of decane.

(vi) One of the most crucial practical issues in the gas sensing field is the influence of the humidity on the sensing parameters. Many studies have assessed the response of sensors to different VOCs, using dry nitrogen as the carrier gas. However, most practical gas sensing applications involve operation under atmospheric conditions with moderate to high humidity back grounds. Here we have studied and compared the performance of the tested PAH-based sensors in dry air $(\sim 5 \% \mathrm{RH})$ and in humidified air ( $40 \%$ and $\sim 80 \% \mathrm{RH})$. All chemiresistors showed a clear increase in resistance when the background $\mathrm{RH}$ levels were increased, while the VOC concentration remained the same (see Figure 2). Most resistance responses to VOCs diluted in $80 \% \mathrm{RH}$ were higher than the response to the same VOCs at $5 \%$ $\mathrm{RH}$, as illustrated by the drastic change of the response patterns in the heat-maps in Figure 2. For example, all PAH/RN-SWCNTs exhibited high responses upon exposure to ethanol, which became more substantial than $80 \%$ RH. Interestingly, as the $\mathrm{RH}$ background increased, the resistance responses to the nonpolar VOCs increased stronger than those of the polar VOCs (excluding mesitylene, which for most PAH films showed the opposite trend).

(vii) In contrast, the QCM sensors gave only very low mass uptakes of water $(<0.3 \%)$, even at fractions of saturated VOC vapor pressure up to $20 \%$. In general, the PAHcoated QCM sensors did show a small increase in response to a specific VOC at a set concentration after a higher humidity background was introduced. However, the heat-maps in Figure 3 reveal that the response patterns of the QCM sensors were 
not dramatically affected by the drastic increase in the RH background level from $5 \%$ to $80 \%$. The only exception was the performance of the PAH-B QCM sensor in an atmosphere of $80 \%$ background $\mathrm{RH}$.

\section{Performance of Cross-reactive Sensors Arrays}

The discriminative power of the PAH/RN-SWCNT chemiresistor array (Array 1; see supporting information, Table S1) and the PAH-QCM sensor array (Array 2) under different RH background levels was tested and compared by means of collective statistical treatment of the sensing responses of the constituent sensors, using both non-supervised (i.e., PCA) and supervised (i.e., DFA) linear multivariate data analysis methods (see Experimental Section). Initially, PCA was used to remove the redundancy of variables and to depict representative maps of the data obtained from both sensor arrays. This is achieved using a linear combination of the original measured variables (i.e., $\Delta R / R_{b}$ for array 1 and $\Delta m / m_{b}$ for array 2), resulting in new uncorrelated variables called principle components (PCs). These PCs allow the reduction of the data dimensionality, facilitating its visualization while retaining as much information as possible.

The main panels of Figures $4(\mathrm{a} \& \mathrm{~b})$ show PCA plots that depict the separation between all nine VOCs examined in the study (see Table 2) at $p_{a} / p_{o}=0.05$ and 0.2 and $\mathrm{RH}=5$ $80 \%$, using arrays 1 and 2, respectively. In the large panel of Figure $4 a$, it can be seen that PC1 (63.3\%), PC2 (22.8\%) and PC3 (5.8\%) account for $91.9 \%$ of the total variance. In the large panel of Figure $4 \mathrm{~b}$, it can be seen that PC1 (76.6\%), PC2 (15.3\%) and PC3 (5.5\%) account for $97.4 \%$ of the total variance. Although it is rather difficult to observe clear clusters and trends in these figures, the separated PCA plots for the three RH levels provide better visualization of the data-set. The side panels of Figure 4a shows that the six PAHfunctionalized RN-SWCNT chemiresistors in array 1 effectively differentiate between the 
various VOCs under 5\% RH background environment. As seen in the PCA panel at 5\% RH, the data points of the polar VOCs (i.e., decanol, octanol, hexanol and ethanol; presented as blue and green hollow triangles) were rather spread over the top-right quarter of the plot. From the same panel, it can be seen also that the points of nonpolar VOC data (i.e., decane, octane, hexane, dibutylether and mesitylene; presented as full orange, violet and reddish shapes) are scattered over (or in proximity) to the bottom-left quarter of the plot. However, increasing the RH background levels to $40 \%$ and $80 \%$ RH blurs this separation slightly.

The PCA plots ascribed to the six PAH-coated QCM sensors in array 2 (see side panels of Figure $4 \mathrm{~b}$ ) show that the data points of the polar VOCs under atmosphere of $5 \% \mathrm{RH}$ are concentrated in the bottom-left quarter of the plot. Additionally, the same plot shows that the nonpolar data points are scattered mostly in the right hemisphere of the plot. While slight overlap of data points is presented under atmospheres of $40 \%$ and $80 \% \mathrm{RH}$, their general distribution does not change compared to that obtained under 5\% RH background.

Interestingly, both array 1 and array 2 exhibit an increase in the PC1 values upon increasing the concentration from $p_{a} / p_{o}=0.05$ to $p_{a} / p_{o}=0.2$ for all examined VOCs. Note that the PC values calculated for each VOC in the separate panels were not identical to the PC values presented in the main figure, because the input data for the models was different.

In order to extensively study and evaluate the classification abilities of the two arrays we employed several DFA models on the data-set. The main panel of Figure 5a shows how array 1 successfully separated the entire group of polar VOCs from the entire group of nonpolar VOCs ( $c f$. Table 2 ) at mixed concentrations between $p_{a} / p_{o}=0.05$ and 0.2 , and under extremely variable RH background levels between $5 \%$ and $80 \% \mathrm{RH}$. The side panels provide separate DFA models for the three RH background levels. Note the CV1 values calculated for each VOC in the separate DFA models were not identical with the CV1 values of the same VOC at the corresponding RH levels in the overall model, because the input data for the 
models was different. The corresponding classification accuracies were derived by leave-oneout cross validation and are listed in Table 3. Despite the extreme variation in concentrations and RH levels within the two compared VOC groups, the six PAH-functionalized RNSWCNT chemiresistive sensors of array 1 achieved a good overall classification accuracy of $73.2 \%$, using only one type of input variable $\left(\Delta R / R_{b}\right)$ from each sensor. As seen in the main panel of Figure 5a, the nonpolar VOCs at the lowest RH level (5\%) formed a tight cluster of data points (full blue squares) that was well separated from the polar data points under $5 \% \mathrm{RH}$ (hollow red squares). The latter gave rise to two separate clusters corresponding to ethanol at different concentrations spreading out to the upper right corner of the DFA map and all other polar VOCs within a tight cluster on the upper left side of the nonpolar cluster. However, with increasing RH background levels (40\% RH and $80 \% \mathrm{RH})$ the clusters of polar and nonpolar VOCs spread out and the data points gradually shifted from the right hemisphere of the DFA map (i.e. positive values of CV1) to the left hemisphere (i.e. towards negative CV1 values). The separate DFA models for the three RH levels further illustrate this trend (see side panels of Figure 5a): The two clusters were very well defined and perfectly separated in an atmosphere of $5 \% \mathrm{RH}$, spreading out and moving closer together while the RH level was increased to $40 \%$ (classification accuracy of polar vs. nonpolar VOCs of $94 \%$; see Table 3), until the clusters started to overlap at $80 \% \mathrm{RH}$, slightly reducing the classification accuracy to $87 \%$.

Array 2 of the six PAH-coated QCM mass-sensitive sensors achieved an even better overall separation between the two groups of polar and nonpolar VOCs (see Figure 5b and Table 3), with an excellent classification accuracy of $92 \%$ after cross validation. The separate DFA analysis at a low background RH of 5\% yielded two clusters that were less well-defined and closer together than the corresponding clusters derived from the resistance responses of array 1. Nevertheless, the intra-cluster spread of data points, the inter-cluster separation, the 
classification accuracy and even the cluster position on the CV1 axis were hardly affected by the strong increase in background RH between the partial models for 5\%, 40\% and $80 \% \mathrm{RH}$ represented in the side panels of Figure 5b. Hence, an array of the PAH-coated QCM sensors such as array 2 could be used for the accurate classification of a VOC's polarity in an atmosphere of extremely variable humidity.

Afterwards, we attempted a more detailed classification of the studied VOCs according to the four different chemical groups that they belong to: alcohols, alkanes, ethers and aromatics (see Table 2). Each group contained different VOCs at concentrations between $p_{a} / p_{o}=0.05$ and $p_{a} / p_{o}=0.2$ at variable background $\mathrm{RH}$ levels between $5 \%$ and $80 \%$. The large panel in Figure 6a displays a three-dimensional DFA model that was derived from the resistance responses of array 1 . As the figure shows, the clusters of the four chemical groups were spread out and overlapped, when analyzing all the data at the different background RH levels together, so that no separation between could be achieved. The classification accuracy of the four groups was almost arbitrary after cross-validation (57\%; see Table 3). This failure resulted from the strong sensitivity of the PAH-functionalized RN-SWCNT sensors to the humidity background. However, when analyzing the data separately at their three separate RH levels, excellent classification results could be achieved. This can be seen in the three side panels of Figure 6a, which demonstrate 2-dimensional DFA maps of the first two canonical variables (CV1 and CV2). At 5\% background $\mathrm{RH}$, each chemical group formed a tight cluster. The four clusters were well-separated from each other with a total classification accuracy of $97 \%$ after cross-validation. Thereby the cluster of the polar alcohols was especially far removed from the other three clusters of the nonpolar groups of alkanes, ethers and aromatics. However, as the humidity background increased, we observed a pronounced increase of the intra-cluster data spread within each chemical group and a decrease in intercluster separation, leading to a decrease in classification accuracy of $94 \%$ for $40 \%$ RH to $80 \%$ 
for $80 \%$ RH (see Table 3 ). The inability to classify all data gathered at varying RH levels together stemmed mainly from a strong shift of the alcohol group (in red) to low values of CV1 and a simultaneous shift of the alkanes group to higher values of CV1.

Figure $6 \mathrm{~b}$ shows the same classification attempt according to the chemical groups, using array 2 of 6 PAH-coated QCM sensors. Excellent and stable values were achieved for the separation accuracy under each humidity background separately (all were above 94\%; see Table 3 and side panels of Figure $6 \mathrm{~b}$ ). Moreover, a very good overall classification was obtained for all the data combined at variable RH levels between 5\% and $80 \% \mathrm{t}(87.1 \%$; see Table 3). Each chemical group clustered separately, however, the mean distance of the aromatic cluster from the other three chemical groups was most pronounced. This is in agreement with the large responses of the separate QCM sensors to the aromatic compounds and can be attributed to the similarity between the VOCs and the fused benzene rings in the PAH cores.

Subsequently, we attempted separating all the nine different VOC examined in this study (see Table 2). An overall separation at RH levels between 5\% and $80 \%$ failed both for array 1 and array 2 , since there were too many separation variables (i.e. VOCs) and not enough predictor variables (i.e. sensing responses). However, we did succeed in separating the clusters of the nine VOCs at each RH background level separately. Figure 7 summarizes the DFA maps for the separate DFA models that were derived from the resistance responses and the QCM mass uptakes. In agreement with our previous results, the best separation was achieved, using the resistance responses of array 1 at 5\% RH (97\% accuracy; see Table 3$)$. The classification accuracy of array 1 decreased slightly and systematically to $89 \%$ for $80 \%$ RH. In contrast, the accuracy of classification of the QCM sensor array increased from $78 \%$ at $5 \% \mathrm{RH}$ to $94 \%$ at $80 \% \mathrm{RH}$. This behavior was expected and can be understood in terms of the lack of compound separation of each separate QCM sensor at low RH and the pronounced 
improvement thereof at increasing RH (see Figures 1\&3). Also in agreement with the results of the separate QCM sensors, the cluster of mesitylene could most easily be distinguished by array 2 , again due to the similarity between mesitylene and the fused benzene rings in the PAH cores.

Finally, we divided array 1 and array 2 into two sub-arrays, in order to gain insight into the role of the polarity of the PAH side-chains on their discrimination power between the separate VOCs. The first sub-array consisted of the PAH-3,4\&5 derivatives which possess alternating hydrophobic alkyl side-chains and hydrophilic side-chains terminated with strong polar functional substituents (ester and carboxyl). The second sub-array consisted of the PAHA,B\&C having only hydrophobic alkyl side-chains (see Table 1). As described above, higher resistance responses and mass uptakes upon exposure to both polar and nonpolar VOCs were measured with the PAH-3,4\&5 sensors than with the PAH-A,B\&C sensors. Nevertheless the higher responses did not yield better discrimination abilities. Table 4 summarizes the classification accuracy for separate DFA models distinguishing between the 4 different polar and the 5 different nonpolar VOCs under the different background RH levels of two subarrays of PAH-functionalized RN-SWCNT chemiresistors and the corresponding two subarrays of PAH-coated QCM sensors. Indeed, the sub-arrays based on PAH-A,B\&C showed superior discriminative ability both for nonpolar and for polar VOCs, despite their lower sensing responses. The same observation was made for the chemiresistor array and the QCM array. The PAH-3,4\&5 derivatives performed reasonably well as chemiresistors, but were clearly inferior to the PAH-A,B\&C molecules as QCM sensing layers. Furthermore, in agreement with the previous results, the classification accuracy for both QCM sub-arrays improved with increasing background $\mathrm{RH}$ level, reaching their maximal values for $80 \% \mathrm{RH}$, while the accuracy of classification with the chemiresistor-sub arrays showed a trend towards lower values in more humid atmospheres. An exception to this rule was the separation of the 4 
polar alcohols, which revealed a maximum at the intermediate $\mathrm{RH}$ value of $40 \%$ for both subarrays. However, this result could very well be an arbitrary outlier. More systematic studies with a larger number of independent measurements would be necessary to confirm these results. The DFA method allows better valuation of the classification abilities of the two arrays and improves their selectivity compared with the PCA results.

\section{SUMMARY AND CONCLUSION}

We have presented a comparative study of the VOCs sensing performance of six customdesigned PAH derivatives with different types of side chains as sensing elements in arrays of chemiresistors and QCM sensors. The PAH derivatives provided good sensitivity and selectivity towards different polar and nonpolar VOCs from the families of alcohols, alkanes, ethers and aromatics under extremely varying humidity conditions $(5-80 \% \mathrm{RH})$, while being quite tolerant to large humidity variations. PAH derivatives having purely nonpolar side chains had lower sensing responses than PAHs with alternating nonpolar and strongly polar side chain termination, but nevertheless showed generally superior classification ability of VOC polarity, chemical class and compound separation when grouped into arrays. Varying humidity levels had opposite effects on arrays of QCM sensors and chemiresistors incorporating the same PAH functional layers: While the resistance response was strongly influenced by background humidity variations, the frequency response was relatively stable over a wide range of humidity levels in the samples, from dry to almost fully humidified atmospheres. We demonstrated that the chemiresistor arrays performed superior at controlled RH levels, especially in dry atmospheres, whereas the QCM arrays were ideally suited for applications under extremely variable background humidity.

Note, however, that the frequency/mass measurements obtained in this work were performed on pure PAH films, while the resistance measurements were performed on PAH/RN-SWCNT bilayers. The reasoning behind facilitating relationships between the two 23 
sets of measurements is that the resonant frequency/mass changes of RN-SWCNT-coated QCM were between 4 to 15 times lower than the values measured by PAH-coated QCM. This observation led us to the postulation that the RN-SWCNT layer has a minor effect on the physical interaction with the organic vapors in relation to the PAH films which act as the sensing layers with the sensors environment.

The results presented here could suggest guidelines governing the use of PAH derivatives in different sensing platforms for a wide range of versatile real-world sensing applications. An investigation the molecules' self-assembly and the resulting morphological properties of the $\mathrm{PAH}$ layers would be of great value for mechanistically rationalizing the sensing properties and optimizing PAH devices for sensing applications. A detailed morphological study of the self-assembled layers presented in this report is underway and will be published elsewhere. However, a true in-depth understanding of the sensing mechanism that would allow flexible adaption of PAH-based sensing platforms requires additional studies of different PAH molecules in which only one property is synthetically altered (e.g. side chain length, functional end groups, and core geometry). 


\section{ASSOCIATED CONTENT}

\section{Supporting Information}

Constituent sensors of the sensor arrays that were used in the current study; and response of PAH-QCM and PAH-4/SWCNT chemiresistor as a function of time at 5\% RH. This material is available free of charge via the Internet at http://pubs.acs.org.

\section{AUTHOR INFORMATION}

Corresponding Author (H.H): hhossam@tx.technion.ac.il

\section{NOTES}

The authors declare no competing financial interest.

\section{ACKNOWLEDGMENTS}

The research leading to these results has received funding from the FP7-Health Program under the LCAOS (Grant 258868), the FP7s ERC grant under DIAG-CANCER (Grant 256639), DFG Priority Program SPP 1355, ESF Project GOSPEL (Grant 09EuroGRAPHENE-FP-001), EU Project GENIUS, and ERC grant on NANOGRAPH. We acknowledge Dr. Ulrike (Mirjam) Tisch, Ms. Alona Byan, Dr. Yair Paska and Ms. Lena Mintz (Technion - IIT) for fruitful discussions. 


\section{REFERENCES}

1. Konvalina, G.; Haick, H. Acc. Chem. Res. 2013, DOI: 10.1021/ar400070m.

2. Broza, Y. Y.; Haick, H. Nanomedicine (Future Medicine) 2013, 8, 785-806.

3. Tisch, U.; Haick, H. MRS Bull. 2010, 35, 797-803.

4. Tisch, U.; Haick, H. Rev. Chem. Eng. 2010, 26, 171-179.

5. Roeck, F.; Barsan, N.; Weimar, U. Chem. Rev. 2008, 108, 705-725.

6. Houser, E. J.; Mlsna, T. E.; Nguyen, V. K.; Chung, R.; Mowery, R. L.; Andrew McGill, R. Talanta 2001, 54, 469-485.

7. Waggoner, P. S.; Craighead, H. G. Lab Chip 2007, 7, 1238-1255.

8. Di Pietrantonio, F.; Cannata, D.; Benetti, M.; Verona, E.; Varriale, A.; Staiano, M.; D'Auria, S. Biosens. Bioelectron. 2013, 41, 328-334.

9. Broza, Y.; Kremer, R.; Tisch, U.; Gevorkyan, A.; Shiban, A.; Best, L. A.; Haick, H. Nanomedicine (New York, NY, US) 2013, 9, 15-21.

10. Haick, H.; Hakim, M.; Patrascua, M.; Levenberg, C.; Shehada, N.; Nakhoul, F.; Abassi, Z. ACS Nano 2009, 3, 1258-1266.

11. Ionescu, R.; Broza, Y.; Shaltieli, H.; Sadeh, D.; Zilberman, Y.; Feng, X.; Glass-Marmor, L.; Lejbkowicz, I.; Müllen, K.; Miller, A.; Haick, H. ACS Chem. Neurosci. 2011, 2, 687-93.

12. Konvalina, G.; Haick, H. ACS Appl. Mater. Interfaces 2011, 4, 317-325.

13. Marom, O.; Nakhoul, F.; Tisch, U.; Shiban, A.; Abassi, Z.; Haick, H. Nanomedicine (Future Medicine) 2012, 7, 639-650.

14. Peled, N.; Barash, O.; Tisch, U.; Ionescu, R.; Broza, Y. Y.; Ilouze, M.; Mattei, J.; Bunn, P. A., Jr.; Hirsch, F. R.; Haick, H. Nanomedicine (New York, NY, US) 2013.

15. Peled, N.; Hakim, M.; Bunn Jr, P. A.; Miller, Y. E.; Kennedy, T. C.; Mattei, J.; Mitchell, J. D.; Hirsch, F. R.; Haick, H. J. Thorac. Oncol. 2012, 7, 1528-1533.

16. Peled, N.; Ionescu, R.; Nol, P.; Barash, O.; McCollum, M.; VerCauteren, K.; Koslow, M.; Stahl, R.; Rhyan, J.; Haick, H. Sens. Actuat. B 2012, 171-172, 588-594.

17. Tisch, U.; Aluf, Y.; Ionescu, R.; Nakhleh, M.; Bassal, R.; Axelrod, N.; Robertman, D.; Tessler, Y.; Finberg, J. P. M.; Haick, H. ACS Chem. Neurosci. 2011, 3, 161-166.

18. Tisch, U.; Billan, S.; Ilouze, M.; Phillips, M.; Peled, N.; Haick, H. CML-Lung Cancer 2012, 5, 107-117.

19. Tisch, U.; Schlesinger, I.; Ionescu, R.; Nassar, M.; Axelrod, N.; Robertman, D.; Tessler, Y.; Azar, F.; Marmur, A.; Aharon-Peretz, J.; Haick, H. Nanomedicine (Future Medicine) 2012, 8, 43-56.

20. Xu, Z. Q.; Broza, Y. Y.; Ionsecu, R.; Tisch, U.; Ding, L.; Liu, H.; Song, Q.; Pan, Y. Y.; Xiong, F. X.; Gu, K. S.; Sun, G. P.; Chen, Z. D.; Leja, M.; Haick, H. Br. J. Cancer. 2013, 108, 941-950.

21. Gutierrez-Osuna, R. IEEE Sens. J. 2002, 2, 189-202.

22. Marco, S.; Gutiérrez-Gálvez, A. IEEE Sens. J. 2012, 12, 3189-3214.

23. Vlachos, D. S.; Skafidas, P. D.; Avaritsiotis, J. N. Sens. Actuat. B 1995, 25, 491-494.

24. Barash, O.; Peled, N.; Tisch, U.; Jr., B. P. A.; Hirsch, F. R.; Haick, H. Nanomedicine (New York, NY, US) 2012, 8, 580-589.

25. Krasteva, N.; Fogel, Y.; Bauer, R. E.; Müllen, K.; Joseph, Y.; Matsuzawa, N.; Yasuda, A.; Vossmeyer, T. Adv. Funct. Mater. 2007, 17, 881-888.

26. Li, B.; Santhanam, S.; Schultz, L.; Jeffries-El, M.; Iovu, M.; Sauvé, G.; Cooper, J.; Zhang, R.; Revelli, J.; Kusne, A.; Snyder, J.; Kowalewski, T.; Weiss, L.; McCullough, R.; Fedder, G.; Lambeth, D. Sens. Actuat. B 2007, 123, 651-660.

27. Peng, H.; Soeller, C.; Vigar, N.; Caprio, V.; Travas-Sejdic, J. Biosens. Bioelectron. 2007, 22, 1868-1873.

28. Regmi, B.; Monk, J.; El-Zahab, B.; Das, S.; Hung, F.; Hayes, D.; Warner, I. J. Mater. Chem. 2012, $22,13732-13741$.

29. Segev-Bar, M.; Shuster, G.; Haick, H. J. Phys. Chem. C 2012, 116, 15361-15368.

30. Shen, G.; Chen, P. C.; Ryu, K.; Zhou, C. J. Mater. Chem. 2009, 19, 828-39.

31. Sysoev, V. V.; Strelcov, E.; Sommer, M.; Bruns, M.; Kiselev, I.; Habicht, W.; Kar, S.; Gregoratti, L.; Kiskinova, M.; Kolmakov, A. ACS Nano 2010, 4, 4487-4494.

32. Wang, J. Electroanalysis 2005, 17, 7-14.

33. Zilberman, Y.; Ionescu, R.; Feng, X.; Müllen, K.; Haick, H. ACS Nano 2011, 5, 6743-6753. 
34. Bachar, N.; Mintz, L.; Zilberman, Y.; Ionescu, R.; Feng, X.; Müllen, K.; Haick, H. ACS Appl. Mater. Interfaces 2012, 4, 4960-65.

35. Xu, X.; Cang, H.; Li, C.; Zhao, Z.; Li, H. Talanta 2009, 78, 711-716.

36. Shin, H.; Yoo, S.; Kim, J.; Kwon, Y. Mol. Cryst. Liq. Cryst. Sci. Technol. A 1997, 295, 137-140.

37. Amal, H.; Ding, L.; Liu, B. B.; Tisch, U.; Xu, Z. Q.; Shi, D. Y.; Zhao, Y.; Chen, J.; Sun, R. X.; Liu, H.; Ye, S. L.; Tang, Z. Y.; Haick, H. Int. J. Nanomed. 2012, 7, 4135-4146.

38. Bayn, A.; Feng, X.; Müllen, K.; Haick, H. ACS Appl. Mater. Interfaces 2013, 5, 3431-3440.

39. Zilberman, Y.; Tisch, U.; Pisula, W.; Feng, X.; Mullen, K.; Haick, H. Langmuir 2009, 25, 54115416.

40. Zilberman, Y.; Tisch, U.; Shuster, G.; Pisula, W.; Feng, X.; Mullen, K.; Haick, H. Adv. Mater. 2010, 22, 4317-4320.

41. Feng, X.; Liu, M.; Pisula, W.; Takase, M.; Li, J.; Müllen, K. Adv. Mater. 2008, 20, 2684-2689.

42. Kastler, M.; Pisula, W.; Wasserfallen, D.; Pakula, T.; Müllen, K. J. Am. Chem. Soc. 2005, 127, 4286-4296.

43. Feng, X.; Pisula, W.; Zhi, L.; Takase, M.; Müllen, K. Angew. Chem. Int. Ed. 2008, 47, 1703-1706.

44. Feng, X.; Pisula, W.; Kudernac, T.; Wu, D.; Zhi, L.; De Feyter, S.; Mu $\square$ llen, K. J. Am. Chem. Soc. 2009, 131, 4439-4448.

45. Feng, X. L.; Marcon, V.; Pisula, W.; Hansen, M. R.; Kirkpatrick, J.; Andrienko, D.; Kremer, K.; Müllen, K. Nature Mater. 2009, 8, 421-426.

46. Hirschberg, J. H.; Brunsveld, L.; Ramzi, A.; Vekemans, J. A.; Sijbesma, R. P.; Meijer, E. W. Nature 2000, 407, 167-170.

47. Lower concentrations than $p_{a} / p_{o}=0.05$ are not stable along the time of the experiment. Higher concentrations than $\mathrm{p}_{\mathrm{a}} / \mathrm{p}_{0}=0.2$ might be associated with condensation of the VOCs on the surface of the sensors ( $c f$. ref. 53). To eliminate these potential problems from our comparative study, the concentratyion in teh current study was limited to $p_{a} / p_{o}=0.05$. .

48. These three RH values are representative examples for low (e.g., 5\%) RH levels that exist usually in closed and well-controlled systems, medium (e.g., 40\%) RH values that exist usually in the atmosphere/environment, and for high (e.g., 80\%) RH values that exist in the environment or in diagnostically-related clinical samples, such as exhaled breath.

49. Brereton, R. G., Chemometrics, Application of Mathematics and Statistics to Laboratory Systems. Ellis Horwood: Chichester, UK, 1990.

50. Charlesworth, M.; Partridge, C.; Garrard, N. J. Phys. Chem. 1993, 97, 5418-5423.

51. Bissell, R. A.; Persaud, K. C.; Travers, P. Phys. Chem. Chem. Phys. 2002, 4, 3482-3490.

52. Topart, P.; Josowicz, M. J. Phys. Chem. 1992, 96, 8662-8666.

53. Paska, Y.; Stelzner, T.; Assad, O.; Tisch, U.; Christiansen, S.; Haick, H. ACS Nano 2011, 6, 335345.

54. Paska, Y.; Stelzner, T.; Christiansen, S.; Haick, H. ACS Nano 2011, 5, 5620-5626.

55. Blackwood, D.; Josowicz, M. J. Phys. Chem. 1991, 95, 493-502.

56. Sherrington, D.; Taskinen, K. Chem. Soc. Rev. 2001, 30, 83-93.

57. Hunter, C.; Lawson, K.; Perkins, J.; Urch, C. J. Chem. Soc. 2001, 5, 651-669.

58. Schon, J. H.; Kloc, C.; Dodabalapur, A.; Crone, B. Appl. Phys. Lett. 2001, 79, 3965-3967.

59. Haynes, W. M., CRC Handbook of Chemistry and Physics 91st ed. CRC Press: Boulder, CO, 2010. 
Table 1: Structural properties of the PAH derivatives

\begin{tabular}{|c|c|c|c|c|c|}
\hline name & core shape & $\begin{array}{l}\text { no. of } C \text { atoms } \\
\text { in the core }\end{array}$ & $\begin{array}{l}\text { side-group } \\
\text { termination }\end{array}$ & $\begin{array}{c}\text { side-group } \\
\text { polarity }\end{array}$ & structure \\
\hline PAH-A & triangular & 60 & $\begin{array}{l}\text { branched } \\
\text { alkyl chain }\end{array}$ & & \\
\hline PAH-B & hexagonal & 42 & $\begin{array}{l}\text { branched } \\
\text { alkyl chain }\end{array}$ & nonpolar & \\
\hline PAH-C & hexagonal & 42 & alkyl chain & & \\
\hline PAH-3 & $\begin{array}{l}\text { semi- } \\
\text { triangular }\end{array}$ & 48 & $\begin{array}{l}\text { alternating } \\
\text { ester (methyl) } \\
\& \text { branched } \\
\text { alkyl chain }\end{array}$ & \multirow{3}{*}{$\begin{array}{l}\text { alternating } \\
\text { strongly polar } \\
\text { and nonpolar } \\
\text { termination }\end{array}$} & \\
\hline PAH-4 & $\begin{array}{l}\text { semi- } \\
\text { triangular }\end{array}$ & 48 & $\begin{array}{l}\text { alternating } \\
\text { carboxyl \& } \\
\text { branched } \\
\text { alkyl chain }\end{array}$ & & \\
\hline PAH-5 & $\begin{array}{l}\text { semi- } \\
\text { triangular }\end{array}$ & 48 & $\begin{array}{l}\text { alternating } \\
\text { carboxyl\& } \\
\text { branched } \\
\text { alkyl chain }\end{array}$ & & \\
\hline
\end{tabular}


Table 2: Physical properties of the VOCs used for the exposure experiments (taken from ref. 59)

\begin{tabular}{|c|c|c|c|c|c|c|c|c|}
\hline VOC & polarity & $\begin{array}{l}\text { chemical } \\
\text { family }\end{array}$ & formula & $\begin{array}{c}\text { molecular } \\
\text { mass } \\
\left(\mathrm{gr}^{\prime} \mathrm{mol}^{-1}\right)\end{array}$ & $\begin{array}{c}\text { vapor } \\
\text { pressure } \\
\left(\mathrm{kpa} @ 20^{\circ} \mathrm{c}\right. \\
)\end{array}$ & $\begin{array}{l}\text { dipole } \\
\text { moment } \\
\text { (debye) }\end{array}$ & $\begin{array}{c}\text { dielectric } \\
\text { constant }\end{array}$ & $\begin{array}{c}\text { density } \\
\left(\mathrm{g} \cdot \mathrm{cm}^{-3}\right)\end{array}$ \\
\hline hexane & \multirow[t]{5}{*}{ nonpolar } & \multirow[t]{3}{*}{ alkanes } & $\mathrm{CH}_{3}\left(\mathrm{CH}_{2}\right)_{4} \mathrm{CH}_{3}$ & 86.23 & 17.6 & 0 & 1.89 & 0.655 \\
\hline octane & & & $\mathrm{CH}_{3}\left(\mathrm{CH}_{2}\right)_{6} \mathrm{CH}_{3}$ & 114.2 & 1.47 & 0 & 1.94 & 0.703 \\
\hline decane & & & $\mathrm{CH}_{3}\left(\mathrm{CH}_{2}\right)_{8} \mathrm{CH}_{3}$ & 142.3 & 1.58 & 0 & 1.98 & 0.730 \\
\hline$d i$-butylether & & ether & {$\left[\mathrm{CH}_{3}\left(\mathrm{CH}_{2}\right)_{3}\right]_{2} \mathrm{O}$} & 130.2 & 0.640 & 0 & 3.1 & 0.769 \\
\hline mesitylene & & aromatic & $\mathrm{C}_{6} \mathrm{H}_{3}\left(\mathrm{CH}_{3}\right)_{3}$ & 120.2 & 0.230 & 0 & 2.4 & 0.864 \\
\hline ethanol & \multirow[t]{5}{*}{ polar } & \multirow[t]{4}{*}{ alcohols } & $\mathrm{CH}_{3} \mathrm{CH}_{2}-\mathrm{OH}$ & 46.07 & 5.95 & 1.69 & 24.3 & 0.789 \\
\hline 1-hexanol & & & $\mathrm{CH}_{3}\left(\mathrm{CH}_{2}\right)_{5}-\mathrm{OH}$ & 102.2 & 0.133 & 1.80 & 13.3 & 0.813 \\
\hline 1-octanol & & & $\mathrm{CH}_{3}\left(\mathrm{CH}_{2}\right)_{7}-\mathrm{OH}$ & 130.2 & 0.114 & 1.76 & 10.3 & 0.824 \\
\hline 1-decanol & & & $\mathrm{CH}_{3}\left(\mathrm{CH}_{2}\right)_{9}-\mathrm{OH}$ & 158.3 & 0.015 & 1.68 & 7.93 & 0.830 \\
\hline water & & water & $\mathrm{H}_{2} \mathrm{O}$ & 18.01 & 2.34 & 1.85 & 80.4 & 1.000 \\
\hline
\end{tabular}

Table 3: Classification accuracy (\%) derived by leave-one-out cross validation for the DFA models represented in Figures 5-7.

\begin{tabular}{|c|c|c|c|c|c|}
\hline \multirow[t]{2}{*}{ DFA model } & \multirow[t]{2}{*}{ sensors array } & \multicolumn{4}{|c|}{ classification accuracy (\%) } \\
\hline & & $5 \% \mathrm{RH}$ & $40 \% \mathrm{RH}$ & $80 \%$ RH & all data \\
\hline \multirow{2}{*}{$\begin{array}{l}\text { differentiating between a group of } \\
\text { polar and a group of nonpolar } \\
\text { VOCs (cf. Figure 5) }\end{array}$} & $\begin{array}{l}\text { array 1: PAH-functionalized } \\
\text { RN-SWCNTs chemiresistors }\end{array}$ & 100 & 94.4 & 86.9 & 73.2 \\
\hline & array 2: PAH-coated QCM & 100 & 100 & 94.4 & 91.6 \\
\hline \multirow{2}{*}{$\begin{array}{l}\text { differentiating between different } \\
\text { chemical families of alcohols, } \\
\text { alkanes, ethers and aromatic ( } c f \text {. } \\
\text { Figure 6) }\end{array}$} & $\begin{array}{l}\text { array 1: PAH-functionalized } \\
\text { RN-SWCNTs chemiresistors }\end{array}$ & 97.2 & 94.4 & 80.6 & 56.6 \\
\hline & array 2: PAH-coated QCM & 94.4 & 98.1 & 94.4 & 87.1 \\
\hline \multirow{2}{*}{$\begin{array}{l}\text { differentiating between the } \\
\text { separate VOCs (decanol, octanol, } \\
\text { hexanol, ethanol, decane, octane, } \\
\text { hexane, dibutylether and } \\
\text { mesitylene; cf. Figure 7) }\end{array}$} & $\begin{array}{l}\text { array } 1 \text { PAH-functionalized RN- } \\
\text { SWCNTs chemiresistors }\end{array}$ & 97.2 & 94.4 & 88.9 & \multirow[t]{2}{*}{$\begin{array}{l}\text { arbitrary } \\
\text { results }\end{array}$} \\
\hline & array 2: PAH-coated QCM & 77.8 & 91.6 & 94.4 & \\
\hline
\end{tabular}


Table 4: Discrimination accuracy between the four different polar VOCs and five different nonpolar VOCs under various background RH levels of two sub-arrays of PAH-functionalized RN-SWCNT chemiresistors (PAH-3,4\&5, which stands for alternating strongly polar and nonpolar side chains; and PAH-A,B\&C that stands for nonpolar side chains alone) and the corresponding two sub-arrays of PAH-coated QCM sensors.

\begin{tabular}{|l|c|c|c|c|c|}
\hline \multicolumn{2}{|c|}{} & \multicolumn{2}{|c|}{ PAH-functionalized RN-SWCNTs } & \multicolumn{2}{c|}{ PAH-coated QCM } \\
\hline group & humidity background & PAH-3,4\&5 & PAH-A,B\&C & PAH-3,4\&5 & PAH-A,B\&C \\
\hline \multirow{3}{*}{$\begin{array}{l}\text { nonpolar } \\
\text { VOCs }\end{array}$} & $5 \% \mathrm{RH}$ & 90 & 100 & 55 & 65 \\
\cline { 2 - 6 } & $40 \% \mathrm{RH}$ & 70 & 95 & 60 & 75 \\
\cline { 2 - 6 } & $80 \% \mathrm{RH}$ & 70 & 85 & 75 & 90 \\
\hline \multirow{3}{*}{$\begin{array}{l}\text { polar } \\
\text { VOCs }\end{array}$} & $5 \% \mathrm{RH}$ & 87.5 & 87.5 & 50 & 87.5 \\
\cline { 2 - 6 } & $40 \% \mathrm{RH}$ & 93.8 & 100 & 68.8 & 87.5 \\
\cline { 2 - 6 } & $80 \% \mathrm{RH}$ & 87.5 & 93.8 & 75 & 100 \\
\hline
\end{tabular}



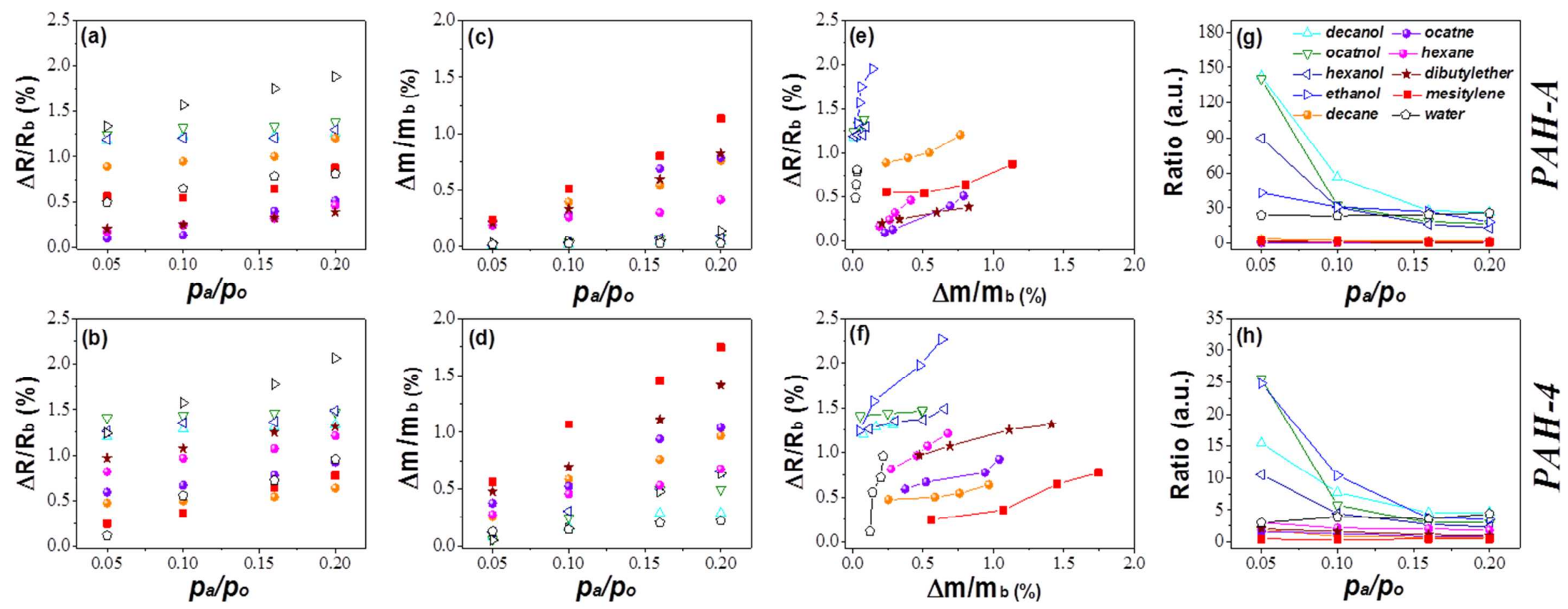

Figure 1: Plots of relative electrical resistance changes $\left(\Delta R / R_{b}\right) v s$. VOC concentration $\left(p_{a} / p_{o}\right)$ for (a) PAH-A, as a representative example for the group of PAH derivatives with only hydrophobic alkyl side-chains; and (b) PAH-4, as a representative example for the group of the PAH derivatives with alternating hydrophobic alkyl side-chains and hydrophilic side-chains. Plots of relative mass changes $\left(\Delta \mathrm{m} / \mathrm{m}_{b}\right) v s$. VOC concentration for (c) PAH-A; and (d) PAH-4. Plots of $\Delta R / R_{b} v s . \Delta m / m_{b}$ for (e) PAH-A; and (f) PAH-4. Plots of the ratio between $\Delta R / R_{b}$ and the $\Delta m / m_{b} v s$. VOC concentration $\left(p_{a} / p_{o}\right)$ for (g) PAH-A; and (h) PAH-4. All data presented in the plots were obtained under 5\% RH background. The error bars of the measurements are smaller than the symbol size. 


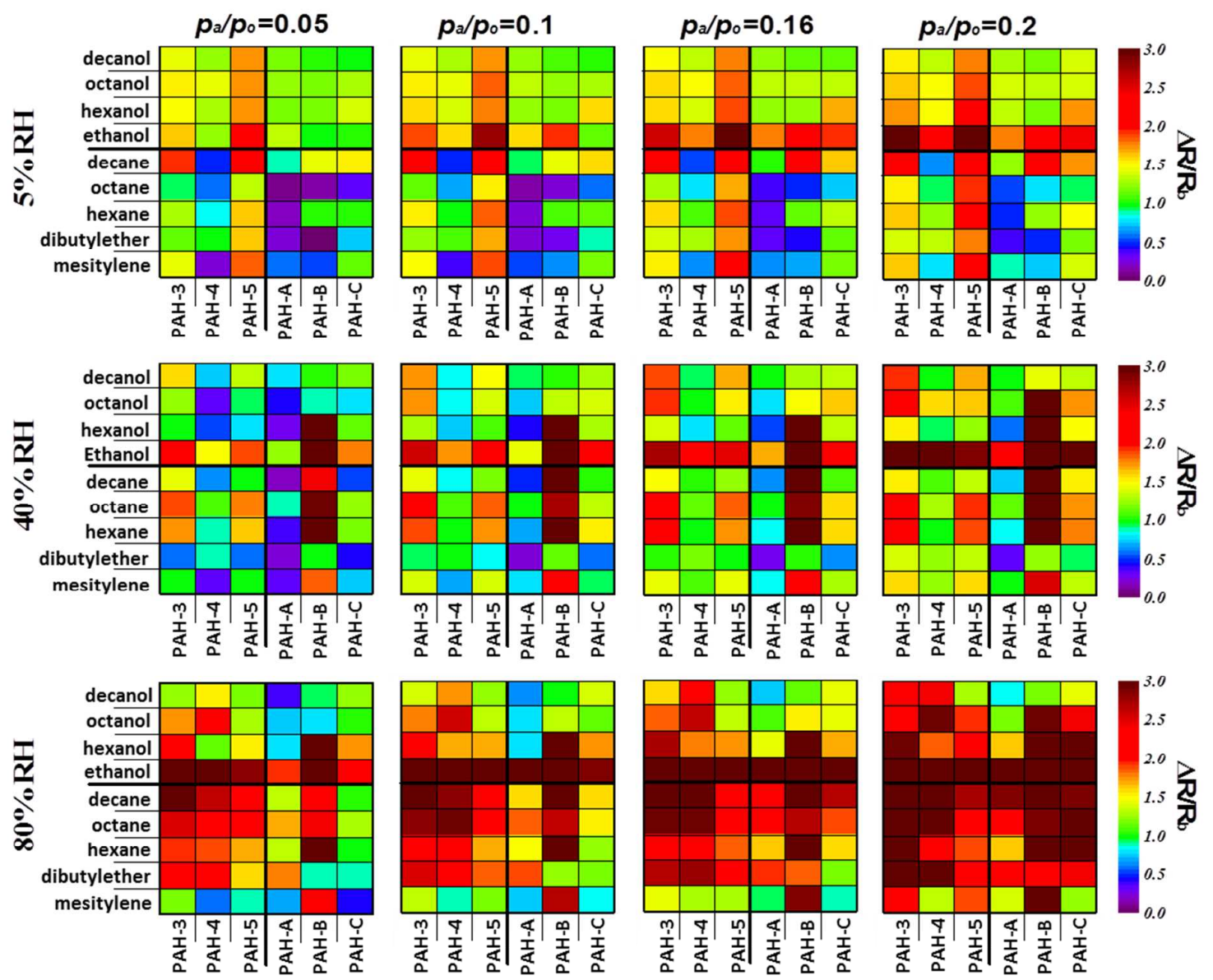

Figure 2: Heat-maps of the relative electrical resistance changes $\left(\Delta R / R_{b}\right)$ of the six constituent PAHfunctionalized RN-SWCNT chemiresistors in array 1 upon exposure to 4 polar VOCs and 5 nonpolar VOCs, at four VOC concentrations (columns): $p_{a} / p_{o}=0.05,0.1,0.16$, and 0.2 ; under three levels of background humidity (rows): $\mathrm{RH}=5 \%, 40 \%$ and $80 \%$. The signal strength is represented by the differences in color. 

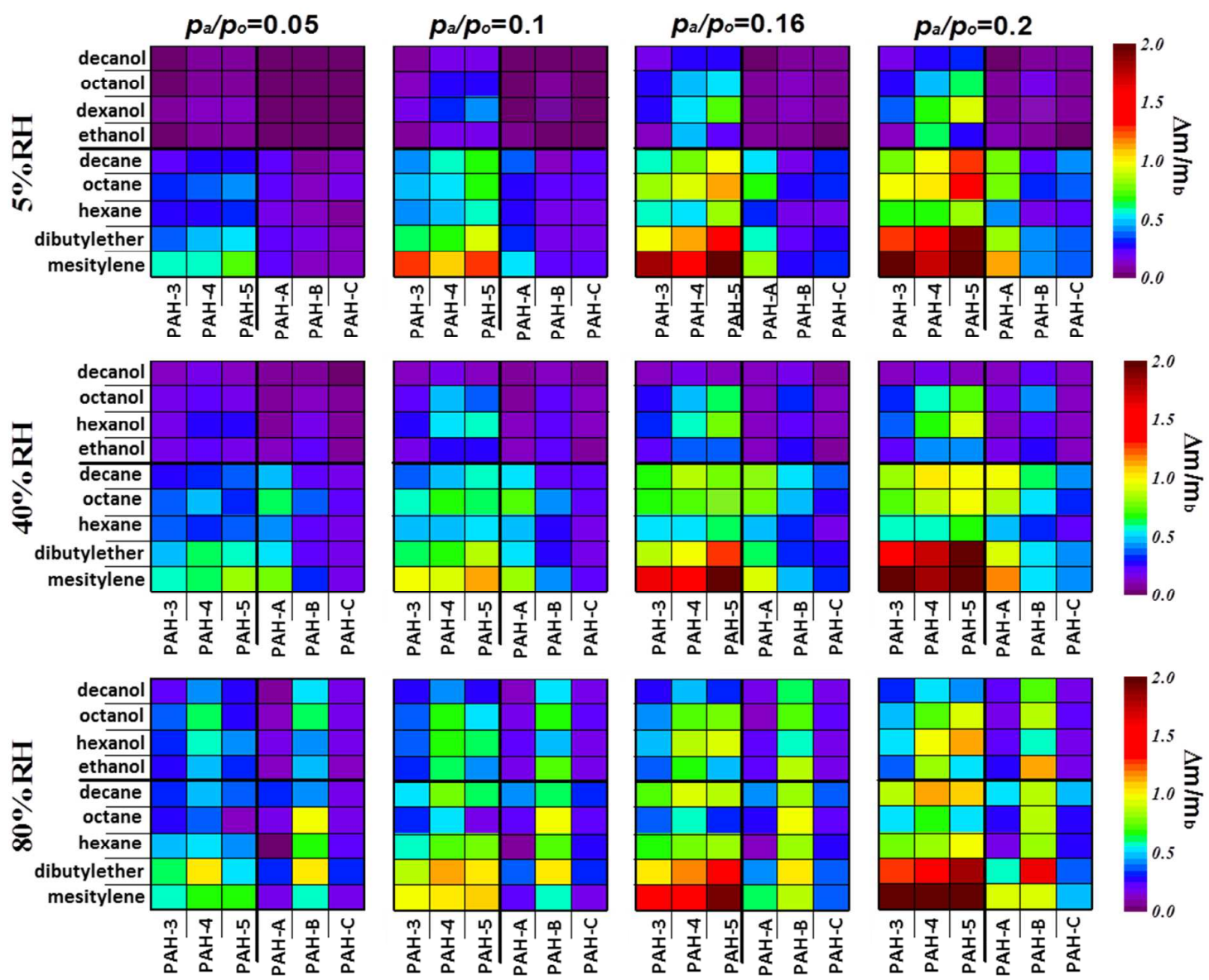

Figure 3: Heat-maps of the relative mass changes $\left(\Delta m / m_{b}\right)$ of the six constituent PAH-coated QCM sensors in array 2 upon exposure to 4 polar VOCs and 5 nonpolar VOCs, at four VOC concentrations (columns): $p_{a} / p_{o}=0.05,0.1,0.16$, and 0.2 ; under three levels of background humidity (rows): $\mathrm{RH}=$ $5 \%, 40 \%$ and $80 \%$. The signal strength is represented by the differences in color. 

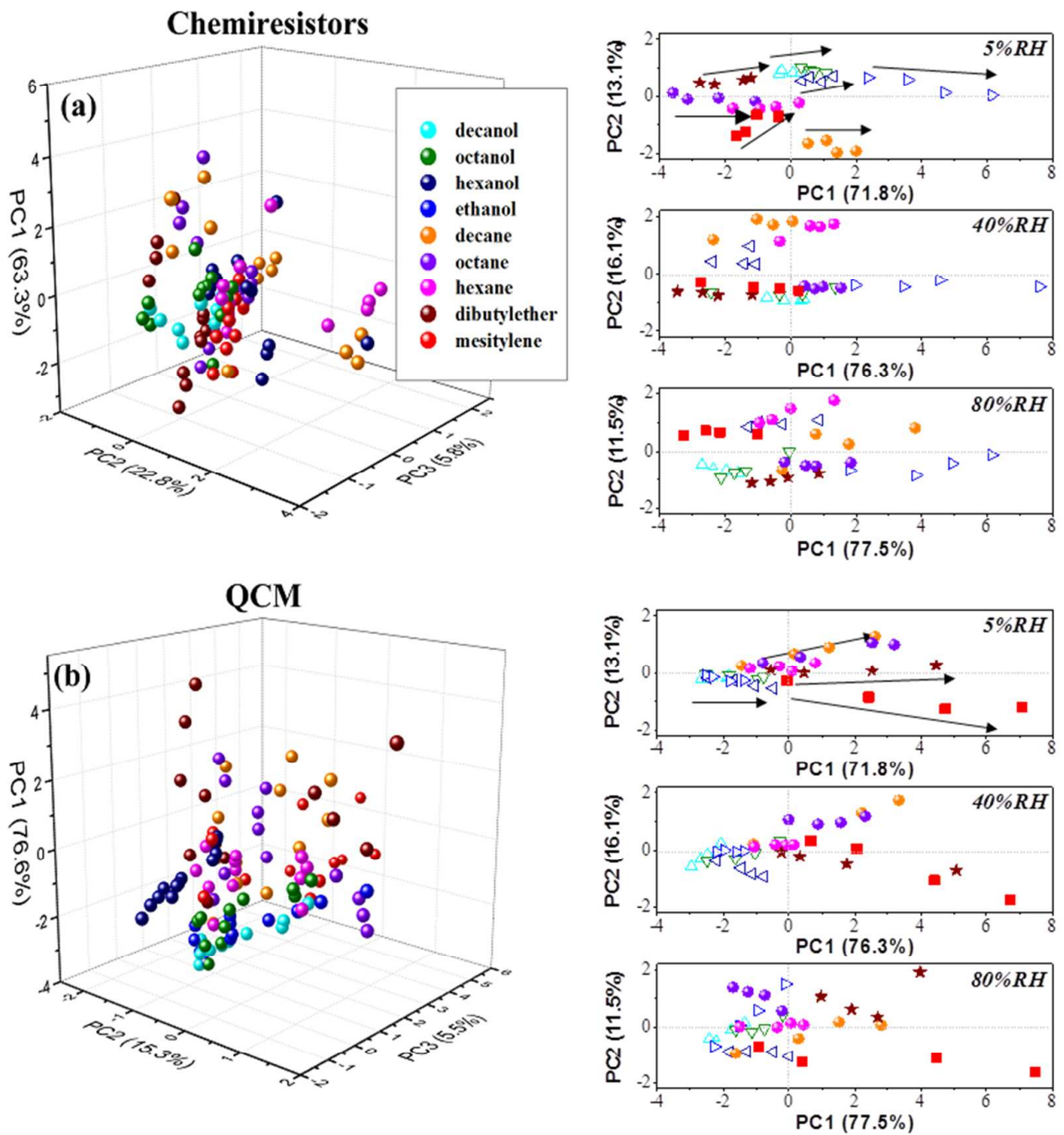

Figure 4: PCA plots separating between the different VOCs examined in the study (decanol, octanol, hexanol, ethanol, decane, octane, hexane, dibutylether and mesitylene, $p_{a} / p_{o}=0.05-0.2, \mathrm{RH}=5-80 \%$ ) using (a) the complete array 1 of six PAH-functionalized RN-SWCNT chemiresistive sensors, and (b) the complete array 2 of six PAH-coated QCM mass-sensitive sensors. The side panels show the PCA plots of separate analysis at the three $\mathrm{RH}$ levels. The arrows direction indicate an increasing concentration from $p_{a} / p_{o}=0.05$ to $p_{a} / p_{o}=0.2$. 

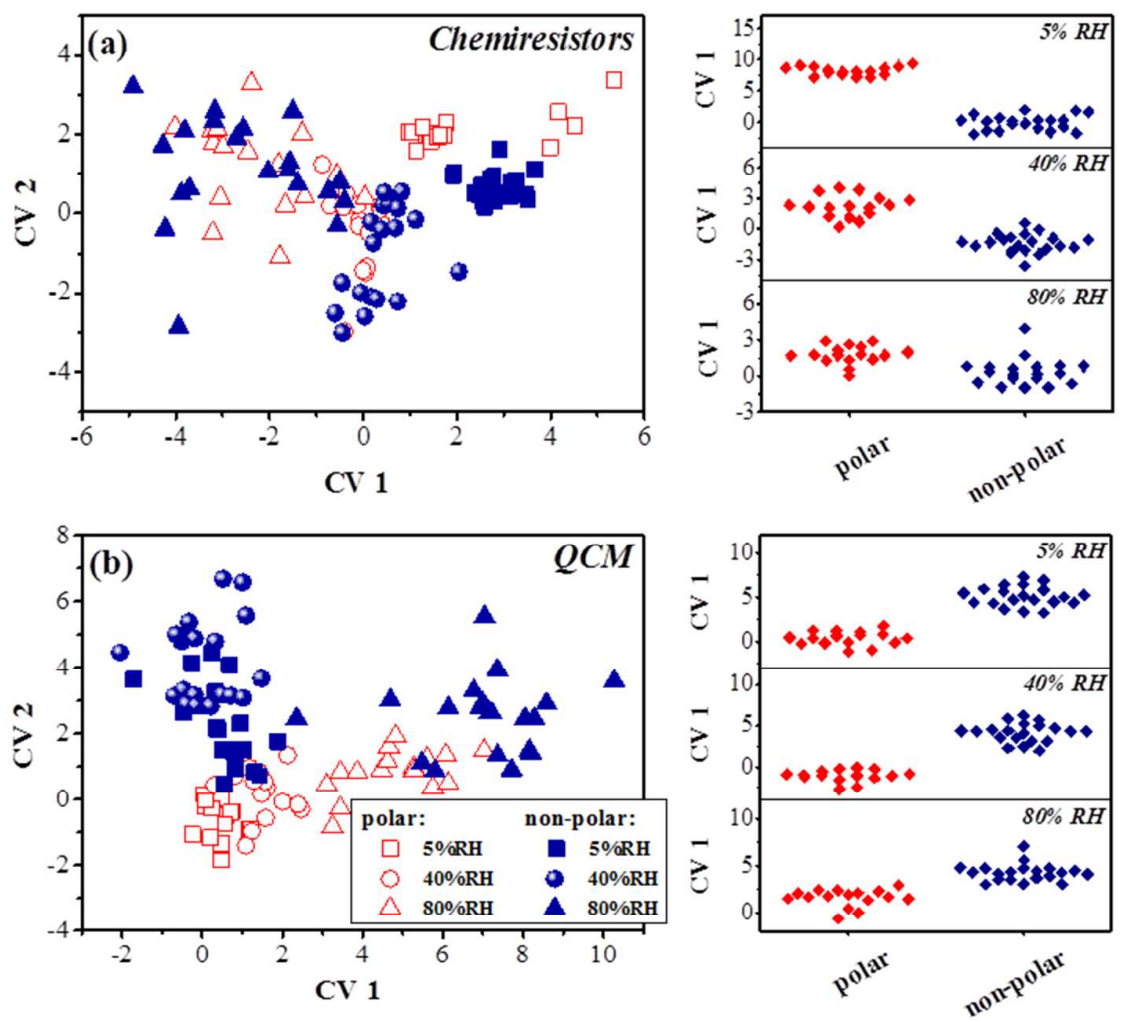

Figure 5: DFA results differentiating between the group of polar VOCs and the group of nonpolar VOCs $(c f$. Table 1$)$ at different concentrations $\left(p_{a} / p_{o}=0.05,0.1,0.16,0.2\right)$ under $5 \%, 40 \%$ and $80 \%$ RH using (a) the complete array 1 of six PAH-functionalized RN-SWCNT chemiresistive sensors, and (b) the complete array 2 of six PAH-coated QCM mass-sensitive sensors. The side panels show the binary DFA maps of separate analysis at the three RH levels. 


\section{Chemiresistors}
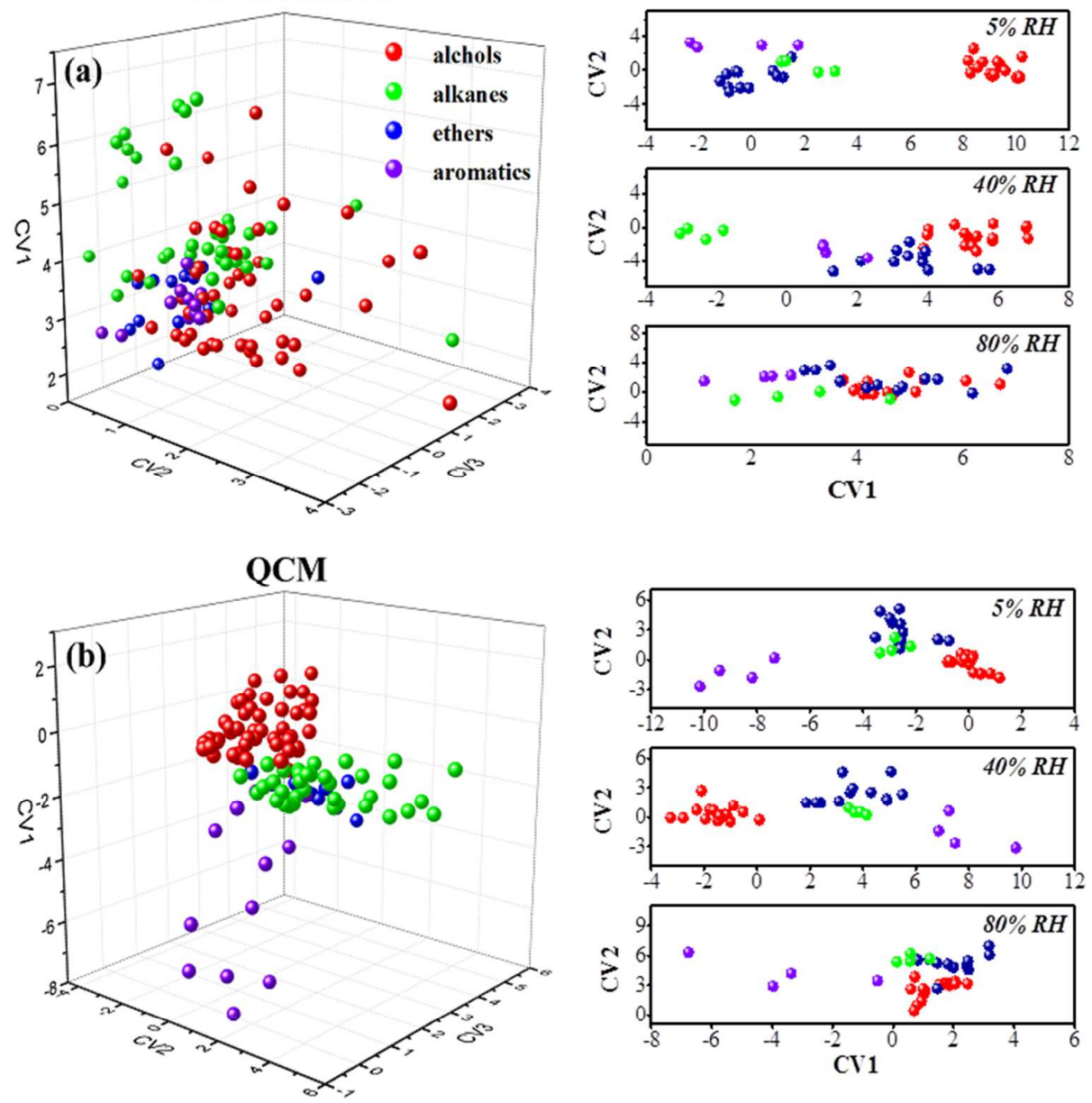

Figure 6: DFA results differentiating between different chemical families of alcohols, alkanes, ethers and aromatics ( $c f$. Table 1) at different concentrations $\left(p_{a} / p_{o}=0.05,0.1,0.16,0.2\right)$ under $5 \%, 40 \%$ and $80 \%$ RH using (a) the complete array 1 of six PAH-functionalized RN-SWCNT chemiresistor, and (b) the complete array 2 of six PAH-coated QCM mass-sensitive sensors. The side panels show the DFA maps of separate analysis at the three RH levels. 


\section{PAH-based QCM}
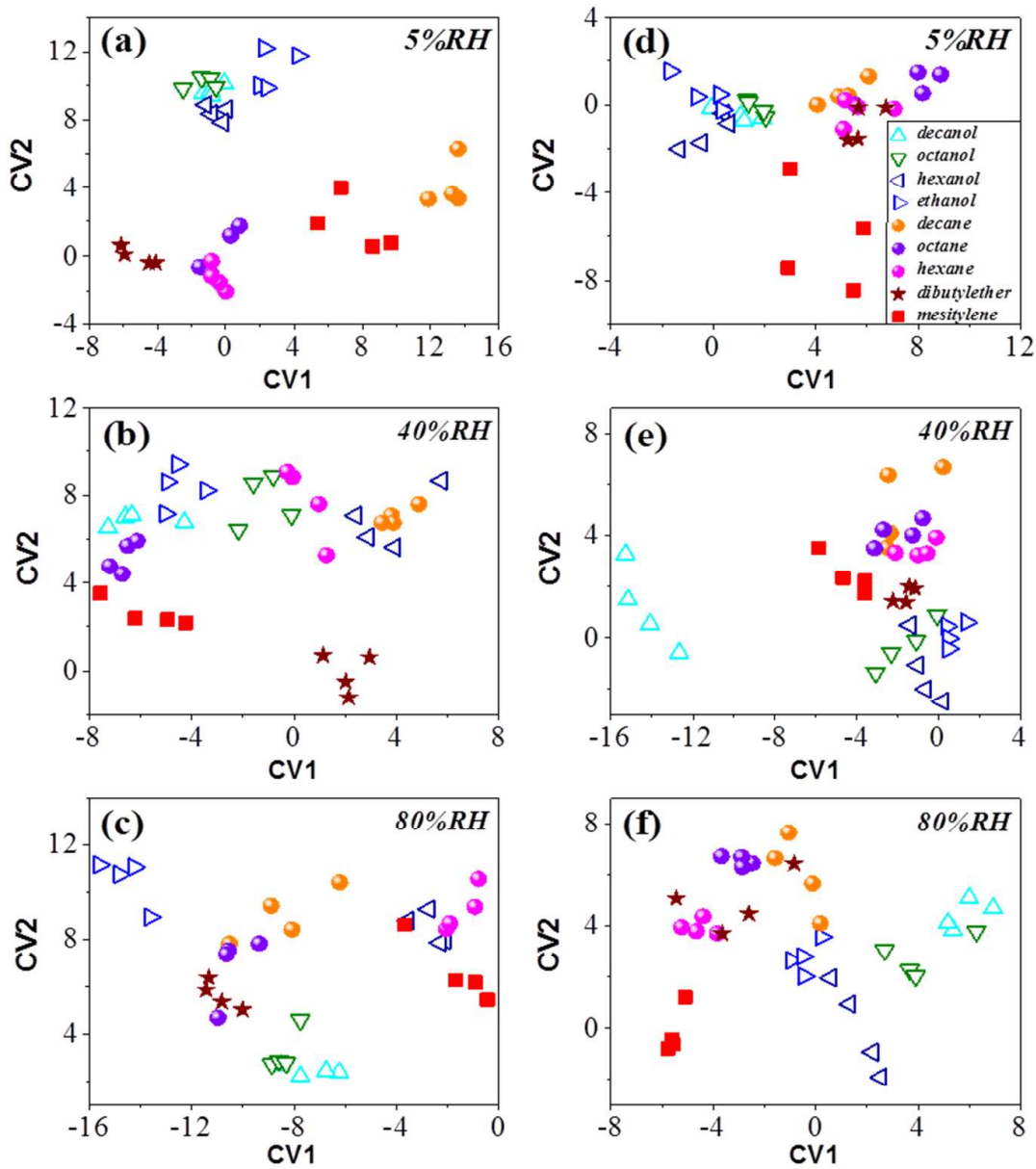

Figure 7: DFA maps of models separating between the different VOCs examined in the study under humidity levels of (a) 5\%, (b) 40\% and (c) $80 \%$ RH using a complete array of 6 PAH-functionalized RN-SWCNT chemiresistor. DFA results of the data measured for the same VOCs under (d) $5 \%$, (e) $40 \%$ and (f) $80 \% \mathrm{RH}$ using a complete array of six PAH-coated QCM mass-sensitive sensors. 


\section{TOC Figure:}

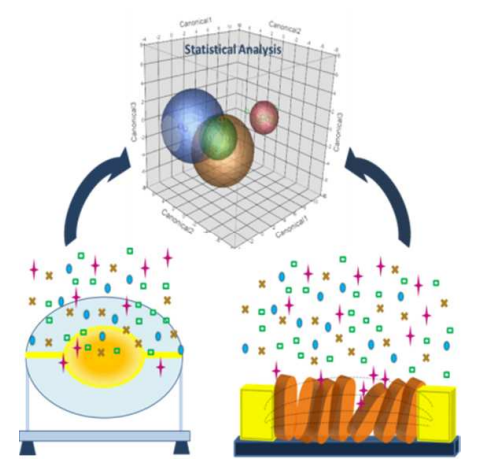

17

18

19

20

21

22

23

24

25

26

27

28

29

30

31

32

33

34

35

36

37

38

39

40

41

42

43

44

45

46

47

48

49

50

51

52

53

54

55

56

57

58

59

60 